\title{
Viscoelastic Parameter Identification of Asphalt Mixture Based on 1stOpt Method
}

\author{
Fuyu Wang ${ }^{1, a}$, Haibin Wei ${ }^{1, b}$ and Ziqi $\mathrm{Li}^{1, \mathrm{c}, *}$ \\ ${ }^{1}$ College of Transportation, Jilin University, Changchun 130022, China \\ awfy@jlu.edu.cn, bweihb@jlu.edu.cn, c535970100@qq.com
}

Keywords: asphalt mixture,1stOpt software, Burgers model.

Abstract. At present, commonly used methods to identify Burgers model parameters have subjectivity shortcomings. This paper adopts 1 stOpt software with unique nonlinear fitting function. The stress-strain-time equation can be obtained according to constitutive equation of Burgers model. Then four parameters of the Burgers model can be obtained by fitting the experimental data directly. Compare with the parameters precision obtained by the subsection fitting method and Lsqcurvefit method and the results show that 1stOpt method has high recognition precision and accuracy.

\section{Introduction}

Back in the 1960s, Monismith ${ }^{[1]}$ applied the classic Boggs model in viscoelastic analysis to viscoelastic analysis of asphalt mixture. Because the classic Boggs model can reflect the viscoelastic properties of asphalt mixture, the Boggs model is still used in the study of viscoelastic mechanical behavior of asphalt mixture ${ }^{[2-7]}$. The recognition accuracy of the Boggs model parameters is an important factor affecting the viscoelastic analysis of asphalt mixture. The data are collected through creep test, and the Boggs model parameters are obtained by corresponding numerical analysis and linear regression. Due to the different methods, the results of the method are different, and the impact on the results of viscoelastic analysis are also significant. This paper will compare 1stOpt method, subsection fitting method and Lsqcurvefit method.

\section{Burgers model parameter identification theory}

Based on viscoelasticity theory ${ }^{[8]}$, different viscoelastic models can be composed of springs which characterize elastic mechanics and dashpots which characterize viscous mechanics. The Burgers model is a four-unit model consisting of two springs and two dashpots. It is composed of a Kelvin element made in parallel by a spring and a dashpot and a Maxwell element made in series by a spring and a dashpot in series. And its constitutive equation is:

$$
\sigma+p_{1} \times \sigma+p_{2} \times q_{1} \times \& \&+q_{2} \times \text {. }
$$

In this equation: $p_{1}=\left(E_{1} \eta_{1}+E_{2} \eta_{1}+E_{1} \eta_{2}\right) / E_{1} E_{2}, p_{2}=\eta_{1} \eta_{2} / E_{2}, q_{1}=\eta_{1}, q_{2}=\eta_{1} \eta_{2} / E_{2}, \quad \sigma$ represents stress and $\varepsilon$ represents strain.

Substitute $\sigma=\Delta(\mathrm{t}) \sigma_{0}$ into the constitutive equation, and the stress-strain equation can be obtained by using Laplace transformation and inverse Laplace transformation:

$$
\varepsilon(t)=\sigma_{0}\left(\frac{1}{E_{1}}+\frac{t}{\eta_{1}}+\frac{1}{E_{2}}\left(1-e^{-\left(-\frac{E_{2}}{\eta_{2}} t\right)}\right)\right) .
$$

Identify the Burgers model parameters, obtain the data of stress-strain-time through creep test, carry on curve fitting according to formula 2, so that the four parameters of the Burgers model can be obtained: $\eta_{1}, \eta_{2}, \mathrm{E}_{1}, \mathrm{E}_{2}$. 


\section{Burgers model common identification methods and 1stOpt method}

Subsection fitting method ${ }^{[9]}$ and Lsqcurvefit method ${ }^{[10]}$ are the most commonly used identification methods at present.

Subsection fitting method: divide the creep curve into three sections, carry on the corresponding curve fitting for the initial segment and the end, then Burgers parameters can be obtained by solving the simultaneous equations. But it's hard to pick the segments and needs a great deal of human trial, which will inevitably produce error. This method also requires high-precision test instruments.

Lsqcurvefit method: use nonlinear fitting function Lsqcurvefit to carry on the curve fitting according to formula 2. But it is hard to give the initial value, so it will require repeated trial and take time and energy.

1stOpt method has a powerful nonlinear fitting function, and can give the initial value on its own, then the optimal solution can be obtained through its unique algorithm. While, other mathematical software must be given an initial value when using iterative method to optimize computation.

\section{Creep test}

\section{Making of samples}

The samples are made of AH-70 asphalt, of which basic performances meet the specifications ${ }^{[11]}$. The mixture gradation is AC-16.The optimal oil-rock ratio is $4.9 \%$ for the Marshall method, and shape the standard specimens at the optimal ratio. Make sure the porosity is at $4 \% \pm 1 \%$.

Table 1 Asphalt mixture gradation table

\begin{tabular}{llllllllllll}
\hline Mesh size[mm] & 19 & 16 & 13.2 & 9.5 & 4.75 & 2.36 & 1.18 & 0.6 & 0.3 & 0.15 & 0.075 \\
\hline Passing rate & 100 & 95 & 88 & 73 & 44 & 29 & 20 & 14 & 10 & 8 & 6 \\
\hline
\end{tabular}

\section{Uniaxial creep test}

The test instrument adopts the British Cooper multi-functional pneumatic asphalt material testing machine, test stress is $500 \mathrm{kpa}$, and the temperature is $50{ }^{\circ} \mathrm{C}$. The test instrument is shown in figure 1 . Before the test starts, the test equipment should be preheated to the test temperature and the test piece should be kept at the test temperature for no less than 4 hours ${ }^{[7]}$. Preload for 30 s first and preload stress is $5 \%$ of load stress. After loading for 3600s, unload for 30s. Test data are automatically collected by the instrument, and derive the test data for subsequent processing after the test is completed. Taking the time as abscissa and the deformation as ordinate,the strain - time relationship of uniaxial creep is shown in figure 2 .
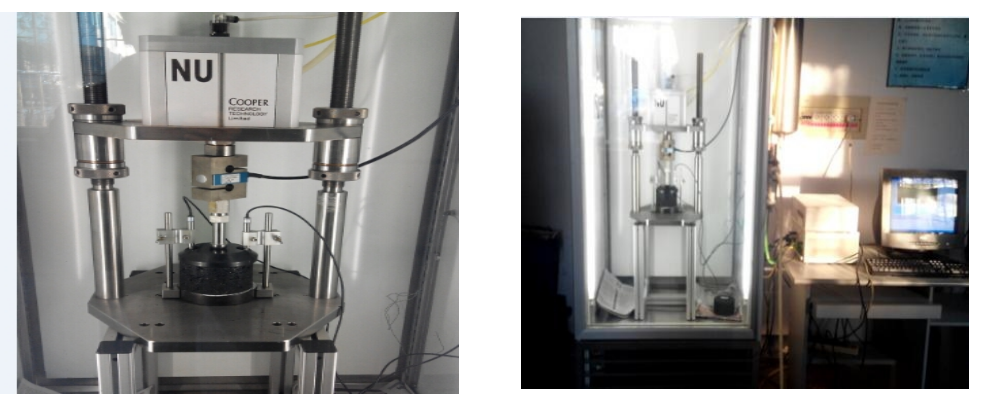

Fig 1 British Cooper testing machine

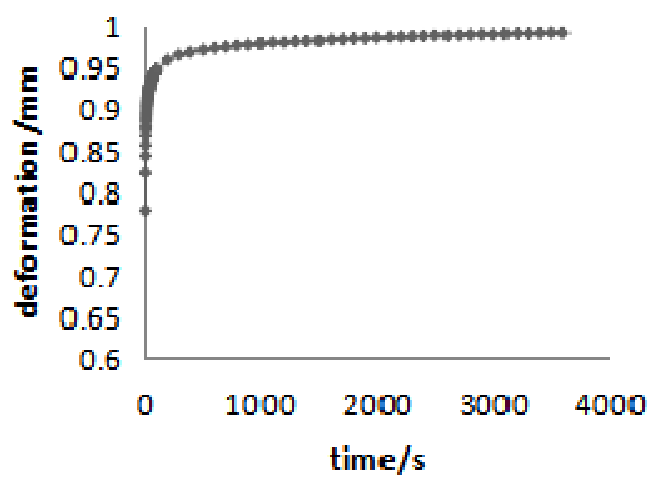

Fig 2 Strain - time curve 


\section{Accuracy analysis of different identification methods}

\section{The recognition process of 1stOpt method parameters}

1stOpt is an excellent software with complete intellectual property, developed independently by the 7D-Soft High Technology Inc. In curve fitting (linear fitting and nonlinear fitting), curve regression and many fields, it occupies the leading position in the world.

In the algorithmic setting of the software interface, the optimization algorithm is: Levenberg Marquardt (LM) + universal global optimization algorithm, and other items are default. Define the code in the codebook: 'Parameter' Define the parameter, 'Variables' Define the variable, 'Function' Define the function of the curve fitting (Equation 2), 'data' define the data from the creep test. After definition of the code, use the shortcut key F9 to run the fit and the software will automatically calculate the output. As the initial value from the 1stOpt software optimization calculation is given randomly, run the calculation for several times and take the result with the largest correlation coefficient as the final result. The fitting diagram of the data from the creep test in this text is shown in figure 4, and the output of the parameter is:

$\mathrm{a} 1=567.39, \mathrm{a} 2=103166195.67, \mathrm{a} 3=5345.89, \mathrm{a} 4=407607.78$,

In the equation, a1, a2, a3, a4 represent E1、 1 1、E2、 $\eta 2$.

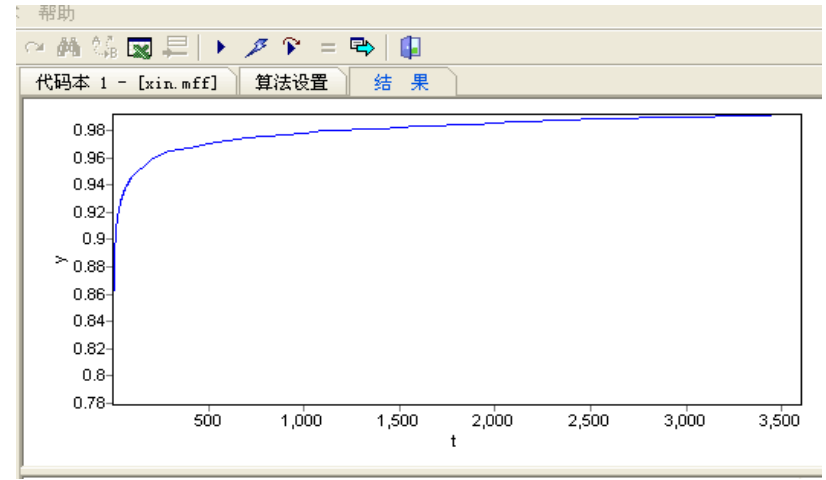

Fig 3 AC-16 asphalt mixture creep test data fitting curve

The process of obtaining parameters of those two methods is described in literature [9] and [10]. The parameters identified by the three methods are listed in table 2 . The result of the subsection fitting method is used as the initial value in the Lsqcurvefit function method.

Table 2 The parameter values identified by the three methods

\begin{tabular}{ccccc}
\hline Identification method & $\mathrm{E}_{1}$ & $\eta_{1}$ & $\mathrm{E}_{2}$ & $\eta_{2}$ \\
\hline Subsection fitting method & 666.67 & 166666666.7 & 2000.0 & 25003.8 \\
Lsqcurvefit method & 647.12 & 166666666.0 & 2823.6 & 28075.0 \\
1stOpt method & 567.39 & 103166195.7 & 5345.9 & 407607.8 \\
\hline
\end{tabular}

\section{Comparison of different methods}

The parameters obtained by the three methods are substituted into the fitting equation of Burgers model (equation 2). Then use MATLAB software data processing functions to calculate the creep deformation data in reverse, while describe the creep test data in the same figure as is shown in figure 
4. In figure 5,the time is the abscissa, the relative error is the ordinate.And in figure 6 , the time is the abscissa, the residual square sum is the ordinate.

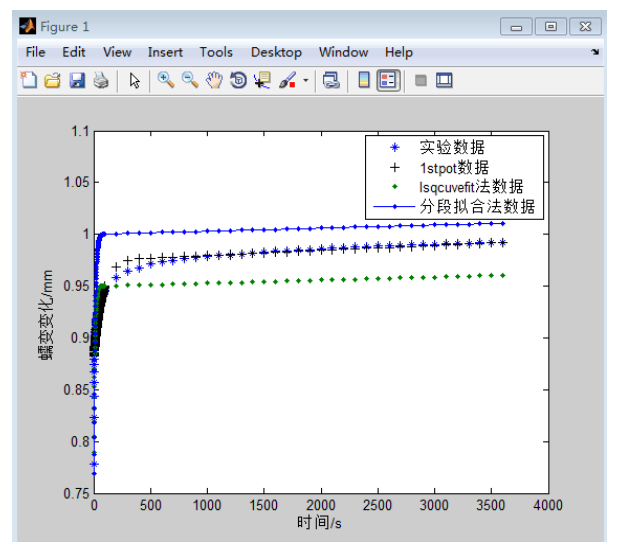

Fig 4 Test data and the inverse data

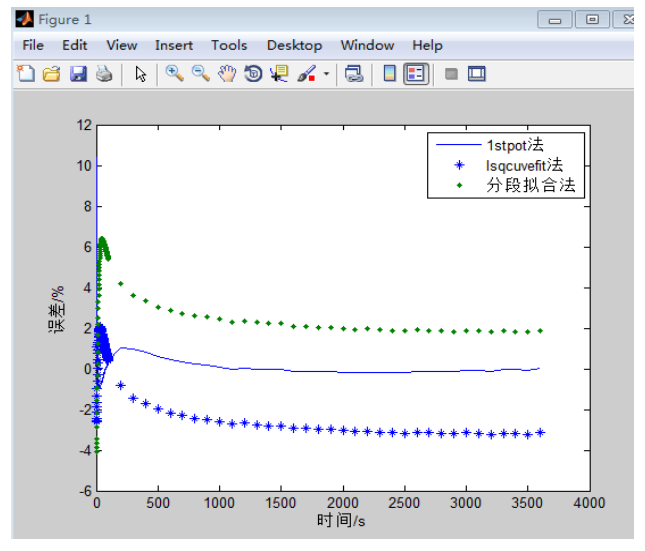

Fig 5 Comparison of the fitting errors

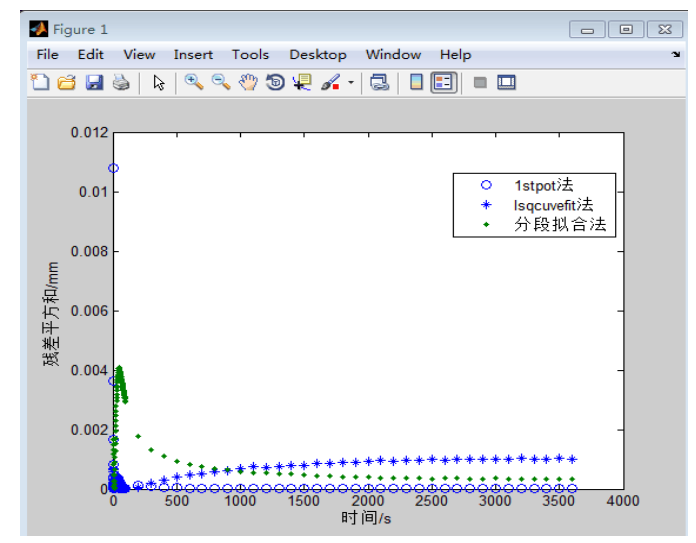

Fig 6 Comparison of residual sum of squares

It can be seen from Fig. 4 that the curves of the three methods are consistent with the trend of the test data curve. The trend of creep deformation in the initial stage changes rapidly, and then tends to grow gently. The 1stOpt software fitting curve is most close to the test data. There is a clear distance between the other two methods and the experimental data points. It is shown that the accuracy of the three methods to identify parameters is different.

As is shown in Fig. 5, the error curve of the subsection fitting method and the lsqcurvefit method increase rapidly in the initial segment, and then there is a faster reduction stage, and finally tend to be gentle.The overall trend of the 1stOpt method is consistent with the previous two, but the trend of the initial segment is slower. This is mainly because in the initial segment,the sudden stress results in uneven force, the accuracy of the test instrument itself is very high, and the human error of the subsection fitting method when processing the data makes the error in the initial segment become larger. The initial value of the lsqcurvefit method is the result of the subsection fitting method. The error of the initial segment is also affected by the result of the subsection fitting method. The 1stOpt method is to fit the experimental data of all stages. Therefore, when the integral data curve fitting is optimal, the error of the initial segment is the smallest. In general, the error curve of 1 stOpt is closest to the $\mathrm{x}$-axis, and the degree of 1stOpt's fitting is the best.

It can be seen from Fig. 6 that the square sum of the residuals of the 1stOpt method is almost coincident with the $\mathrm{x}$-axis and the other two curves are over it. Therefore, the 1stOpt method has the best fitting degree, and the curve of subsection fitting method has an intersection with the lsqcurvefit curve. The fitting of the two methods can be evaluated by overall square sum of the residuals. For the 
sum of squares of the overall residual, 1stOpt method $(0.0099)<1$ sqcurvefit method $(0.0483)<$ subsection fitting method ( 0.3087 ). And corresponding to fitting precision, 1 stOpt method > lsqcurvefit method $>$ subsection fitting method.

\section{Conclusion}

In this paper,we can get the Burgers model parameter by using the unique and powerful nonlinear fitting function of 1stOpt software. With a standard of relative error and residual sum of square,the subsection fitting method, Lsqcurvefit function method and the 1stOpt method are comparatively analyzed. Results from the comparison indicate that the 1stOpt method obtains parameters with the highest accuracy. The 1stOpt method overcomes the shortcomings of the subsection fitting method of human handling factors and the inconvenience of non-high-precision instruments, and difficulty of the Lsqcurvefit function method to obtain reasonable initial value. It is easy to operate and promote.

\section{Acknowledgements}

The authors sincerely appreciate for financial support of the National Natural Science Foundation of China under Grants Nos.51578263, Transportation Science \& Technology Program of Jilin Province.

\section{References}

[1] Feng Lin, The research on the static and dynamic creep model of asphalt mixture under uniaxial pressure[D],Changsha University of Science \& Technology, 2008.

[2] Burgers J M. Mechanical consideration-model systems phenomendogical theories of relation and of viscosity[R]. New York: First Report on Viscosity and Plasticity Nordeman Publishing Company, 1935.

[3] Christensen R M. Theory of viscoelasticity: an introduction[M].New York:Academic Press, Inc. 1982.

[4] Shashidhar N, Shenoy A. On using micromechanical models to describe dynamic mechanical behavior of asphalt mastics[J]. Mechanics of Materials, 2002,34 (10) : 657-669.

[5] Abbas A R. Simulation of the micromechanical behavior of asphalt mixtures using the discrete element method[D]. Pullman: Washington State University, 2004.

[6] Yang Datian, Xia Wenjun, Yang Xiwu, Creep experiments evaluating the flowing deformation of the asphalt treated permeable base in high temperature condition[J],Journal of Chongqing Jiaotong University(Natural Science),2008,27(5):758-752.

[7] Liu Yu, You Zhan-ping. Determining Burger's model parameters of asphalt materials using creep-recovery testing data[C]//Proceedings of the Symposium on Pavement Mechanics and Materials at the Inaugural International Conference of the Engineering Mechanics Institute, 2008:26-36.

[8] Zhang Xiaoning, Principle and application of viscoelastic mechanics of asphalt and asphalt mixture[M], China Communication Press, 2006.1

[9] Tian Li, Hu Xiaguang, Liu Yu, Sha Aimin, Sectioned linear fitting method of parameters for asphalt mastics viscoelastic Burgers model[J],Journal of Traffic and Transportation Engineering,2007.7(3):66-69 
[10] Chen Jingyun, Zhou Changhong, Wang Zheren, Data processing and viscoelastic computation for creep test of asphalt mixture[J], Journal of Southeast University(Natural Science Edition), 2007.37(6):1091-1095.

[11] Ministry of Transport of the People's Republic of China, Technical specification for construction of highway asphalt pavements(JTG F40-2004), 2005 